\title{
Orthonormalization of Substrate and Guided Modes in Effective Resonator Model of Dielectric Multilayer Structure ERRATUM
}

\author{
A. RUDZIŃSKI* \\ Institute of Microelectronics and Optoelectronics \\ Warsaw University of Technology \\ Koszykowa 75, 00-665 Warszawa, Poland
}

This note contains an erratum to [A. Rudziński, Acta Phys. Pol. A 112, 505 (2007)].

PACS numbers: 99.10.- $\mathrm{x}, 42.70 . \mathrm{Qs}$

The integral (6) in [1] does not vanish for $\boldsymbol{q}=\boldsymbol{k}_{*}$, therefore the value of $\tilde{F}_{k \epsilon}$ for guided modes, given by (24) in [1], is incorrect. The correctly calculated value is

$$
\tilde{F}_{k \epsilon}= \begin{cases}4 \pi^{2} \int_{-\infty}^{\infty} \mathrm{d} z n^{2}(z) \phi_{k_{*} \epsilon}^{*}(z) \phi_{k \epsilon}(z), & \text { if } \rho_{\epsilon}(\boldsymbol{k}) \neq 0, \\ 0, & \text { if } \rho_{\epsilon}(\boldsymbol{k})=0,\end{cases}
$$

where

$$
\begin{aligned}
& \int_{-\infty}^{\infty} \mathrm{d} z n^{2}(z) \boldsymbol{\phi}_{k_{*} \epsilon}^{*}(z) \phi_{k \epsilon}(z)=\frac{n_{(\mathrm{L})}^{2} u_{\mathrm{L} k \epsilon}^{2}}{2\left|k_{z}^{(\mathrm{L})}\right|}+\frac{n_{(\mathrm{R})}^{2} u_{\mathrm{R} k \epsilon}^{2}}{2\left|k_{z}^{(\mathrm{R})}\right|} \\
& \quad+\sum_{-N_{\mathrm{L}}<j<N_{\mathrm{R}}} n_{(j)}^{2} L_{z}^{(j)}\left\{\left[u_{j k \epsilon}^{2} \exp \left(\mathrm{i} k_{z}^{(j)} L_{z}^{(j)}\right)+v_{j k \epsilon}^{* 2} \exp \left(-\mathrm{i} k_{z}^{(j)} L_{z}^{(j)}\right)\right]\right. \\
& \left.\quad \times \boldsymbol{e}_{k_{*}(j) *} \boldsymbol{e}_{k^{(j)} \epsilon} \frac{\sin \left(k_{z}^{(j)} L_{z}^{(j)}\right)}{k_{z}^{(j)} L_{z}^{(j)}}+2 u_{j k \epsilon} v_{j k \epsilon}^{*} \boldsymbol{e}_{k^{(j) *} \epsilon}^{*} \boldsymbol{e}_{k^{(j)} \epsilon}\right\}
\end{aligned}
$$

\section{References}

[1] A. Rudziński, Acta Phys. Pol. A 112, 505 (2007).

*e-mail: arudzins@poczta.onet.pl 